\title{
Image restoration with spatially variable PSF
}

\author{
Paolo Ciliegi ${ }^{a}$, Andrea La Camera ${ }^{b}$, Laura Schreiber $^{a}$, Michele Bellazzini $^{a}$, Mario Bertero $^{b}$, \\ Patrizia Boccacci ${ }^{b}$, Emiliano Diolaiti $^{a}$, Italo Foppiani ${ }^{a}$, Matteo Lombini ${ }^{a}$, Davide Massari $^{c}$, \\ Paolo Montegriffo ${ }^{a}$, Margherita Talia ${ }^{c}$ \\ ${ }^{a}$ INAF - Osservatorio Astronomico di Bologna, Via Ranzani, 1, 40127 Bologna, Italy; \\ ${ }^{b}$ Dipartimento di Informatica, Bioingegneria, Robotica e Ingegneria dei Sistemi (DIBRIS), \\ Università di Genova, Via Dodecaneso 35, 16145, Genova, Italy; \\ ${ }^{c}$ DIFA - Università di Bologna, Viale Berti Pichat 6/2, 40127 Bologna, Italy.
}

\begin{abstract}
We present a method for the restoration of astronomical images obtained with Adaptive Optics (AO) systems. In order to maximize the scientific return from $\mathrm{AO}$ data and, in general, from the data of the next generation telescopes, we developed a restoration method based on deconvolution for the de-blurring of images degraded by a spatially variable PSF. The deconvolution method is based on a partition of the image domain in partially overlapping sub-domains where the PSF can be assumed to be space invariant. The software, called Patch, is written in IDL language and is freely distributed to the community. Here we report a general description of the method and of its graphical interface. The potentiality of the Software Patch have been tested on two completely different astrophysical scenarios: a crowded stellar field and an extended galaxy. Despite the very conservative assumptions made on the Point Spread Function (assumed to be strongly variable across the field of view), we obtained good results in terms of image reconstruction both for the stellar (point-like) case and for the extended galaxy.
\end{abstract}

Keywords: Adaptive Optics, Image processing, Data analysis, Space-Variant Deconvolution, Software Patch

\section{INTRODUCTION}

The future 40 meter class telescopes (E-ELT, ${ }^{1} \mathrm{TMT}^{2}{ }^{2} \mathrm{GMT}^{3}$ ) require AO to fully achieve their scientific goals. However, while the $\mathrm{AO}$ systems correct atmospheric turbulence in real time increasing the energy concentration of the Point Spread Function (PSF), they introduce a spatial variation of the PSF itself across the field of view.

To achieve a more uniform correction on a larger Field of View (FoV), a new technique called Multi-Conjugate Adaptive Optics (MCAO) has been developed. ${ }^{4}$ This technique has been already demonstrated on sky by MAD ${ }^{5}$ on the VLT and by GeMS ${ }^{6}$ on Gemini and it has been planned for the future E-ELT ${ }^{7}$. However, to exploit the full power of MCAO the residual PSF variations across the field have to be considered and modeled anyway. The MCAO systems are relatively rare in the present telescopes, but they will be the ordinary systems in the next generation telescopes (like the E-ELT and the TMT).

In order to maximize the scientific return from $\mathrm{AO}$ data and, in general, from the data of next generation telescopes, we developed a restoration method based on deconvolution for the de-blurring of images degraded by a spatially variable PSF.

An accurate correction of the AO PSF spatial dependence is in fact a fundamental issue in presence of extended objects where a variable blur across the imaged field makes it difficult to perform accurate morphological studies. The implementation of image restoration is also interesting when applied to crowded stellar fields like globular clusters, where a drastic reduction of the crowding effect can be achieved, leading to very interesting improvements also in resolved stellar populations science.

The deconvolution method is based on a partition of the image domain in partially overlapping sub-domains where the PSF can be assumed to be approximately space invariant. In each sub-domain the deconvolution is

Further author information: Send correspondence to Paolo Ciliegi: E-mail: paolo.ciliegi@oabo.inaf.it, Telephone: +39 0512095743

Adaptive Optics Systems IV, edited by Enrico Marchetti, Laird M. Close,

Jean-Pierre Véran, Proc. of SPIE Vol. 9148, 91482O - (c) 2014 SPIE

CCC code: $0277-786 X / 14 / \$ 18 \cdot$ doi: $10.1117 / 12.2055914$

Proc. of SPIE Vol. 9148 91482O-1 
performed by means of an efficient method also providing boundary effect correction. The complete deconvolved image is obtained as a mosaic of the results in non-overlapping sub-domains.

In this paper we report a description of the new deconvolution method, including a description of the Graphical User Interface and the results obtained applying this method on two completely different astrophysical scenarios: a crowded stellar field and an extended galaxy.

\section{METHOD AND SOFTWARE PATCH}

In this section we briefly describe the software, that we called 'Patch', and the method used by the software for space-variant deconvolution.

\subsection{General introduction}

The method we use was recently proposed by some of the authors of this paper ${ }^{8}$ and is based on the sectioning approach introduced by several authors ${ }^{9-12}$. The novelty with respect to the previous versions of this method is the use of a simple and natural approach for accurate boundary effect correction ${ }^{11,12}$ and of an efficient deconvolution method ${ }^{11-13}$.

The method is based on the assumption that the space-variant PSF is smoothly varying over the FoV so that it can be represented by space invariant PSFs over suitable sub-domains (also called patches). Therefore the starting point is a set of $K \times K$ samples of the PSF, with centers in points $\mathbf{n}_{\mathbf{1}}, \mathbf{n}_{\mathbf{2}}, \ldots, \mathbf{n}_{\mathrm{K}^{2}}$ of the image domain. If these points form a suitable uniform grid, then the image domain can be partitioned into $K \times K$ non-overlapping sub-domains, one for each PSF. If standard deconvolution methods are applied to these domains, then the results are affected by boundary effects due both to discontinuities at the boundary introduced by the periodic continuation intrinsic in FFT and to the variation of the PSFs in the adjacent domains. In order to reduce these effects, one first extends the sub domains in order to obtain an overlapping of the adjacent ones, this overlapping being mainly controlled by the extent of the PSFs. Next suitable methods for the reduction of boundary effects in image deconvolution must be used.

The Software Patch is written in IDL, it is made of the main routine (patch.pro) and of several sub-routines contained in a library. Patch can be used directly from its Graphical User Interface (GUI), described in the following section, as well as from the IDL's prompt. In the latter mode, a set of parameters previously prepared (and saved as a 'sav' file) is provided as optional input of the starting command of the software. A Readme.txt file and an online Tutorial are provided together with the package.

As mentioned above, the input of the method and therefore of the software is the image (including the background) to be deconvolved and a set of PSFs, as well as other information such as the sky background, the read-out noise and gain of the CCD.

\subsection{The Graphical User Interface}

The GUI of Patch is organized in three tabs (or panels), each one for a specific task: input, deconvolution, and output. In the following figures, we show some screenshots representing the reconstruction of a simulated image of Hubble Space Telescope (HST) before COSTAR correction*. A complete analysis of the results of this reconstruction can be found in a forthcoming paper ${ }^{8}$.

- Input - In this panel the user can load both the input image and the set of PSFs as FITS files. The loaded image is shown in the graphical area and some options are available on a pop-up menu: zoom, scale and colormap can be changed for a more comfortable visualization. Then the user can choose the number of sub-domains that must be the same number of the input PSFs, i.e. $K \times K$. At this point the overlap value of adjacent domains can be chosen. Even if a further overlap will be automatically added by the software, we suggest to use here a positive number (depending on the PSF extension). More details on the overlap are described in the forthcoming paper ${ }^{8}$. Finally, the PSFs can be loaded on the GUI. Both a cube of PSFs

\footnotetext{
*obtained via anonymous ftp from ftp.stsci.edu in the directory
}

/software/tables/testdata/restore/sims/starcluster/ 
(contained in a unique file) and a set of different files can be uploaded. In the latter case, the filenames must agree with the numbering of the sub-domains (first sub-domain is labelled with 0 and corresponds to the lower-left one on the image. Then numbers grow first to right and then to top).

- Deconvolution - In the second panel, the user must set all the deconvolution parameters and can start deconvolution process. Information such as sky background and CCD camera are set on the left part of this panel. Then, the user can choose between two different deconvolution methods: the well known RichardsonLucy $(\mathrm{RL})^{14,15}$ and the Scaled Gradient Projection (SGP) ${ }^{16}$. With the assumption of Poisson statistics for the data ${ }^{17}$, the two deconvolution methods consist in the minimization of the so-called data-fidelity function (or Kullback-Leibler divergence) given by

$$
J_{0}(\mathbf{f})=\sum_{\mathbf{m} \in S}\left\{\mathbf{g}(\mathbf{m}) \log \frac{\mathbf{g}(\mathbf{m})}{(\mathbf{K} * \mathbf{f}+\mathbf{b})(\mathbf{m})}+(\mathbf{K} * \mathbf{f}+\mathbf{b})(\mathbf{m})-\mathbf{g}(\mathbf{m})\right\},
$$

where $\mathbf{g}$ is the piece of the image corresponding to the current sub-domain, $\mathbf{b}$ is the estimation of the sky background that must not have been subtracted from the image, $\mathbf{f}$ is the unknown object, and $\mathbf{K}$ is the PSF of the current sub-domain (as mentioned before, the deconvolution is performed in each sub-domain).

Both methods are extended with the boundary effect correction ${ }^{18,19}$. The two algorithms are iterative and, except in the case of point-wise objects, early stopping of the iterations is required for obtaining sensible solutions of the restoration problem. Four stopping rules are available for the SGP algorithm, as shown in the following table. The stopping rule \#1 is also available for the RL method.

\begin{tabular}{|l|l|}
\hline Name & Stopping rule \\
\hline \#1: iter > MAX ITER & if $(k>$ MAX_ITER $)$ then stop the iterations \\
\#2: distance of successive iterations & || $\mathbf{f}^{(k)}-\mathbf{f}^{(k-1)}\|<\tau *\| \mathbf{f}^{(k)} \|$ OR \#1 \\
\#3: data-fidelity function & $\left.\mid J_{0}\left(\mathbf{f}^{(k)}\right)-J_{0}\left(\mathbf{f}^{(k-1)}\right)\right)|<\tau *| J_{0}\left(\mathbf{f}^{(k-1)}\right) \mid$ OR \#1 \\
\#4: discrepancy principle & $\frac{2}{N} J_{0}\left(\mathbf{f}^{(k)}\right)<\tau$ OR \#1 \\
\hline
\end{tabular}

In the table, $\mathbf{f}^{(k)}$ is the reconstructed object at $k$-th iteration, $J_{0}\left(\mathbf{f}^{(k)}\right)$ is the data-fidelity function defined in Eq. 1, $N$ is the total number of pixels of $\mathbf{f}$, and $\tau$ is a user-defined tolerance of the stopping rules \#2-\#4. Choose \#3 in order to push the algorithm to convergence. In the case of \#4, called Discrepancy principle, $\tau$ should be a given number close to 1. More information can be found in Bertero et al. $(2010)^{20}$.

Finally, a maximum number of iterations must be chosen in order to avoid infinite loop of the selected algorithm. This number can be the same for each sub-domain or can be chosen in different ways. In Fig. 2 we show the complete set of parameters for the mentioned example.

By pressing the big button "run deconvolution", the algorithm is launched and the program waits for its end. A message printed in the IDL prompt informs the user when a sub-domain is done and the following begins the deconvolution (the elapsed time is also shown). Depending on the working dimensions, on the number of sub-domains, and, above all, on the computer, it might take from a few seconds to several minutes to obtain the final result. A mosaic of the reconstructed sub-domains forms the output image.

- Output - In the last part of the GUI the results of the deconvolution process are displayed and saved. The reconstructed object can be shown (the same visualization options of the input panel are also available here) and saved as a FITS file. The header of the file can be shown and is updated with all the information gathered on the GUI (and possibly useful in further analysis). The so-called residual map can be also shown and saved (as FITS file again). The residual map, defined by

$$
R\left(\mathbf{f}^{(k)}\right)=\frac{\mathbf{g}-\left(\mathbf{K} * \mathbf{f}^{(k)}+\mathbf{b}\right)}{\sqrt{\mathbf{K} * \mathbf{f}^{(k)}+\mathbf{b}}},
$$

contains statistical information about the reconstructed object and its histogram can be also shown. A "perfect" reconstruction should have a mean value equal to zero and variance equal to 1 . In Fig. 3 we show the last part of the GUI, that concludes the reconstruction process. 


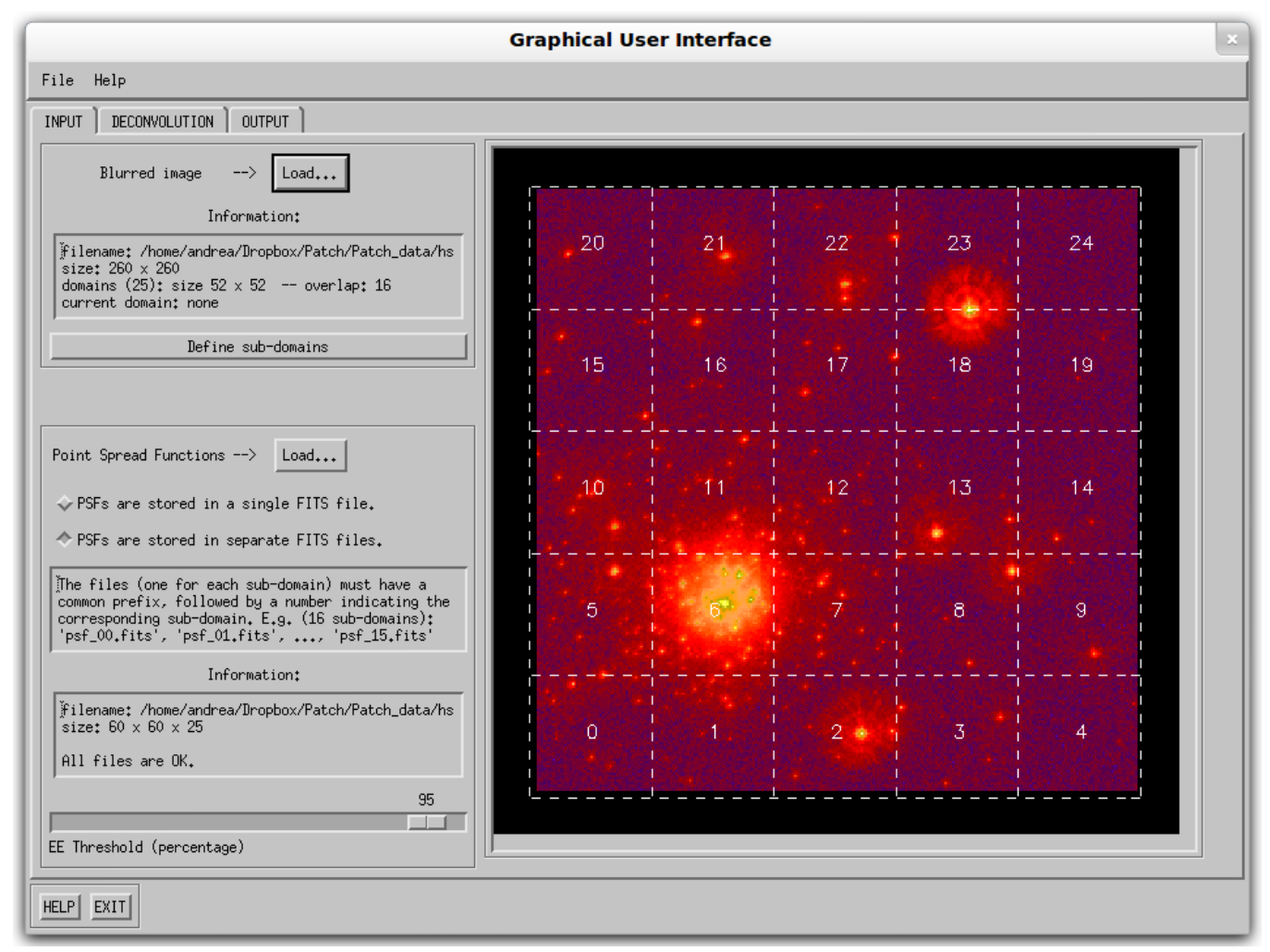

Figure 1. Software Patch - GUI - In the first panel the input of the image and of the PSFs are set.

All the parameters set in the GUI can be saved within an IDL's ".sav" file and can be used later, reloading them for a new deconvolution of the same data, or for an "offline" deconvolution, as mentioned above. This can be useful, for example, in a remote use of IDL/Patch or in a multiple execution of different deconvolutions on the same machine. In this case, supposing to have already set the parameters (and stored in a file "mytest.sav"), the deconvolution can be executed by entering the following command at the IDL prompt:

PATCH > patch, 'mytest.sav'

A menu and a button bar complete the GUI. A tutorial is available online through the Help menu and button.

\subsection{Availability of the code}

The Software Patch is freely distributed and can be downloaded from the software section of the website: http://www . airyproject.eu

\section{APPLICATION TO SCIENCE CASES}

AO data are often characterized by complex PSF, sometimes strongly variable across the FoV. This spacial variability is particularly evident when the $\mathrm{AO}$ system uses only one reference star for the atmospheric turbulence measurement. In this panorama, AO images offer an interesting test-bench for the proposed deconvolution method. In literature, other authors ${ }^{21,22}$ propose the space-variant deconvolution as a necessary tool for the exploitation of $\mathrm{AO}$ corrected images.

The potentiality of the Software Patch has been tested using simulated images of two completely different astrophysical scenarios: point-like sources in a crowded stellar field and an extended extragalactic source. During our data reduction, we considered different PSF grids relative to an increasing number of sub-domains (from $3 \times 3$ to $15 \times 15$ ) in order to trace the dependency of the reconstruction goodness on the PSF knowledge.

In the next sections we describe how the images have been simulated, the PSF models used (both in terms of shape and space-variance) and the results obtained on point-like and extended sources. 


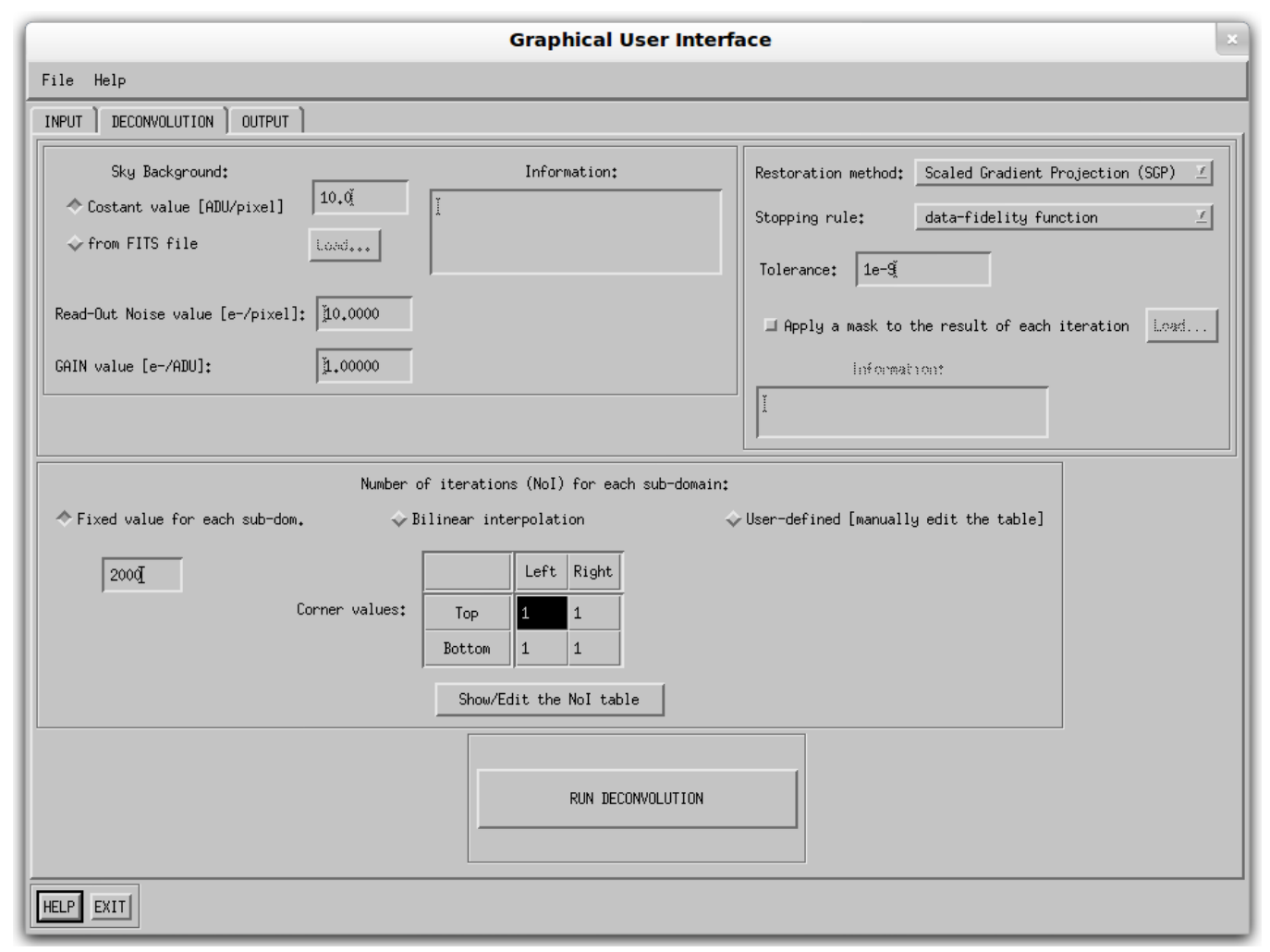

Figure 2. Software Patch - GUI - In the second panel deconvolution parameters such as sky background, CCD data, reconstruction algorithm, stopping rule and total number of iterations to be executed are set. The big button "run deconvolution" starts the process.

\subsection{Image simulation and PSF model}

As first step, we simulated to acquire our frames through a 8.2 meters telescope, equipped with a Single Conjugate AO system. The instrumental parameters that we adopted are very similar to the characteristics of the PISCES Infrared Camera with the Large Binocular Telescope Adaptive Optics System ${ }^{23}$. We used a Read-out noise of 20 $e^{-}$(considering a gain $\left.=1 e^{-} / \mathrm{ADU}\right)$, a $60 \%$ Quantum Efficiency in $J$ band and a dark current of $\sim 0.1 e^{-} / \mathrm{sec}$. We considered a $1024 \times 1024$ pixels detector with a pixel scale of $0.02^{\prime \prime} /$ pixel, corresponding to a field of view $(\mathrm{FoV})$ of $\sim 21.5 \times 21.5$ arcsec. Both the science cases were simulated in the $J$ filter, having central wavelength $=1.27 \mu \mathrm{m}$. We assumed the near-IR sky background measured at Mount Graham ${ }^{24}(\sim 15.8$ mag in $J$ band). The number of individual frames and their exposure times have been adapted in order to reach an acceptable SNR avoiding saturation of any star. The total exposition time has been set equal to 1 hour for the stellar case and 3 hours for the extragalactic case. Details about the two science cases are reported in the next dedicated Sections (3.2 and 3.3).

At a first approximation, the SCAO residual PSF can be modeled as the combination of different analytical components such as Airy disks, Moffat 2D, Lorentzian or Gaussian 2D functions + numerical residuals ${ }^{25}$. In order to simulate images with a continuous space-variant PSF, we adopted a pure analytical model. In this way, it is possible to describe the variation of each PSF parameter across the FoV. The adopted model is the combination of two main components: a diffraction limited core and a seeing limited halo. The AO correction is in fact only partial and does not provide a full compensation for the atmospheric turbulence, causing part of the source flux to remain in a 'non-corrected' larger halo. Each component is described by a Moffat 2D function. A deeper description of the adopted model is reported in the following:

- Diffraction limited Core: Moffat with a radial variation with respect to the Guide Star (GS) direction. The Moffat rotation angle depends on the GS position in the field, in order to reproduce the typical SCAO 


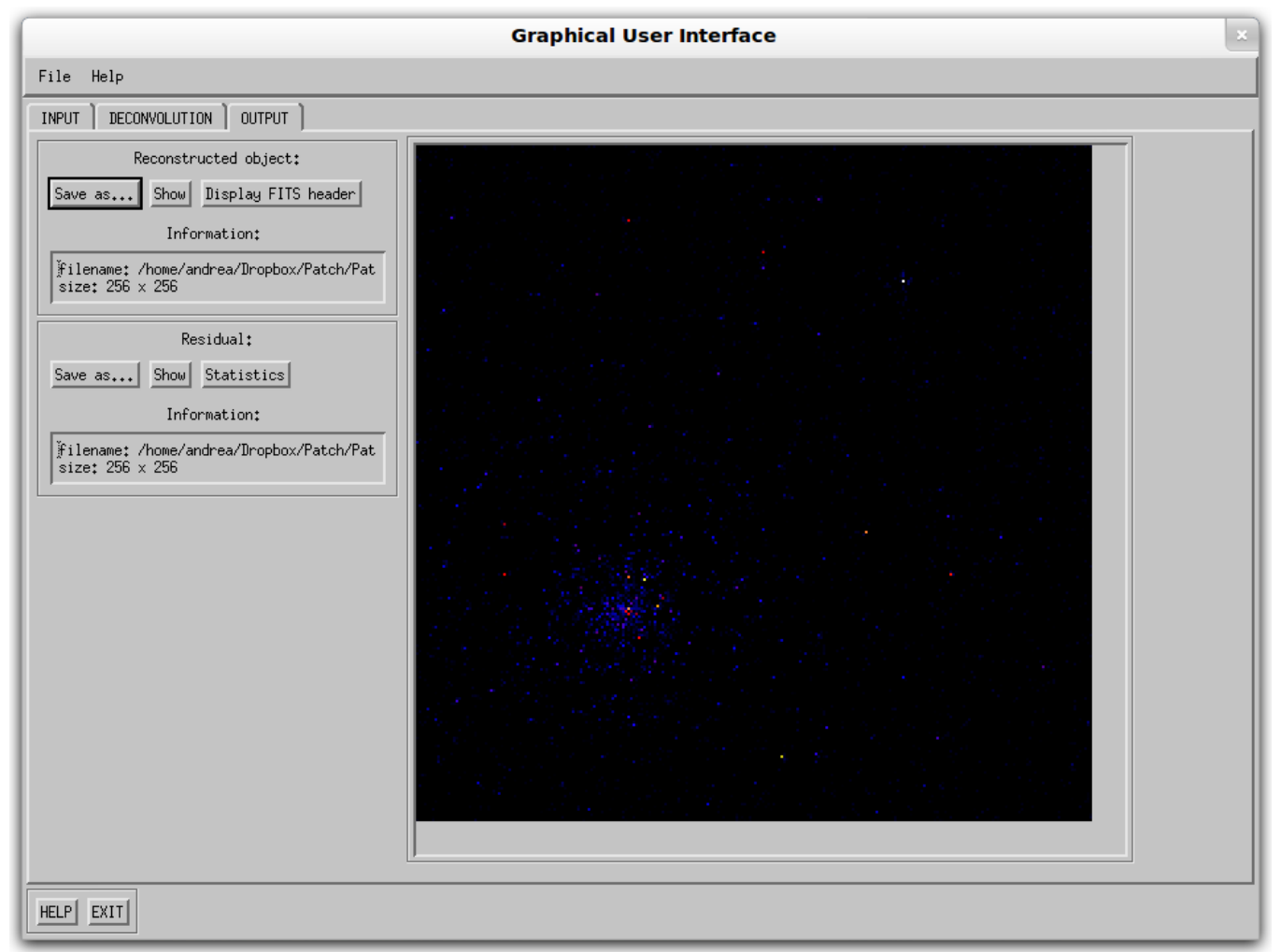

Figure 3. Software Patch - GUI - In the third panel the reconstructed object and the statistical information on the results can be visualized and saved on FITS files.
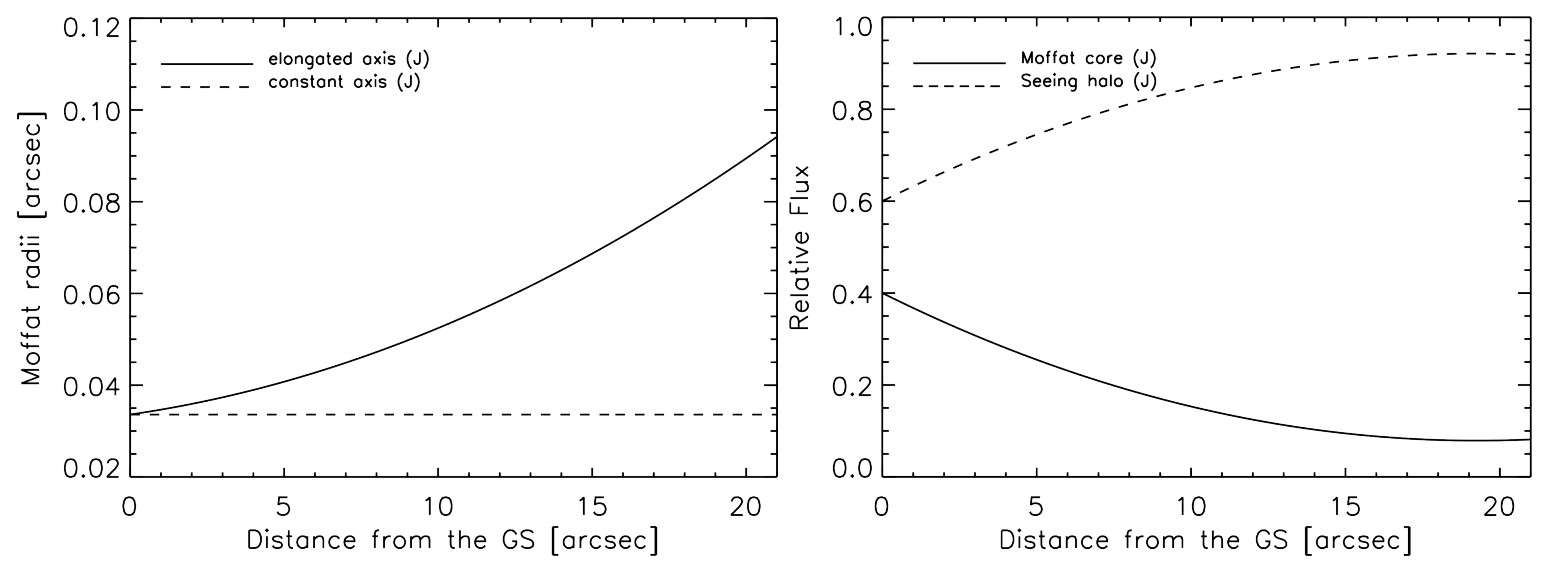

Figure 4. Left panel: Variation of the Moffat Core component half-light radii in $J$ band across the FoV with respect to the GS distance in arcseconds. The continuous line refer to the elongated half-light radiul, i.e. the one pointing toward the GS (positioned at coordinates $[0,0]$ ). The dashed line refer to the non-elongated radius, i.e. pointing in the direction orthogonal to the elongation direction. The non-elongated radius have been considered constants in this simplified model. Right panel: Variation with respect to the GS distance of the relative Flux contained in each of the two PSF components: Core (continuous lines) + Halo (dashed lines). 

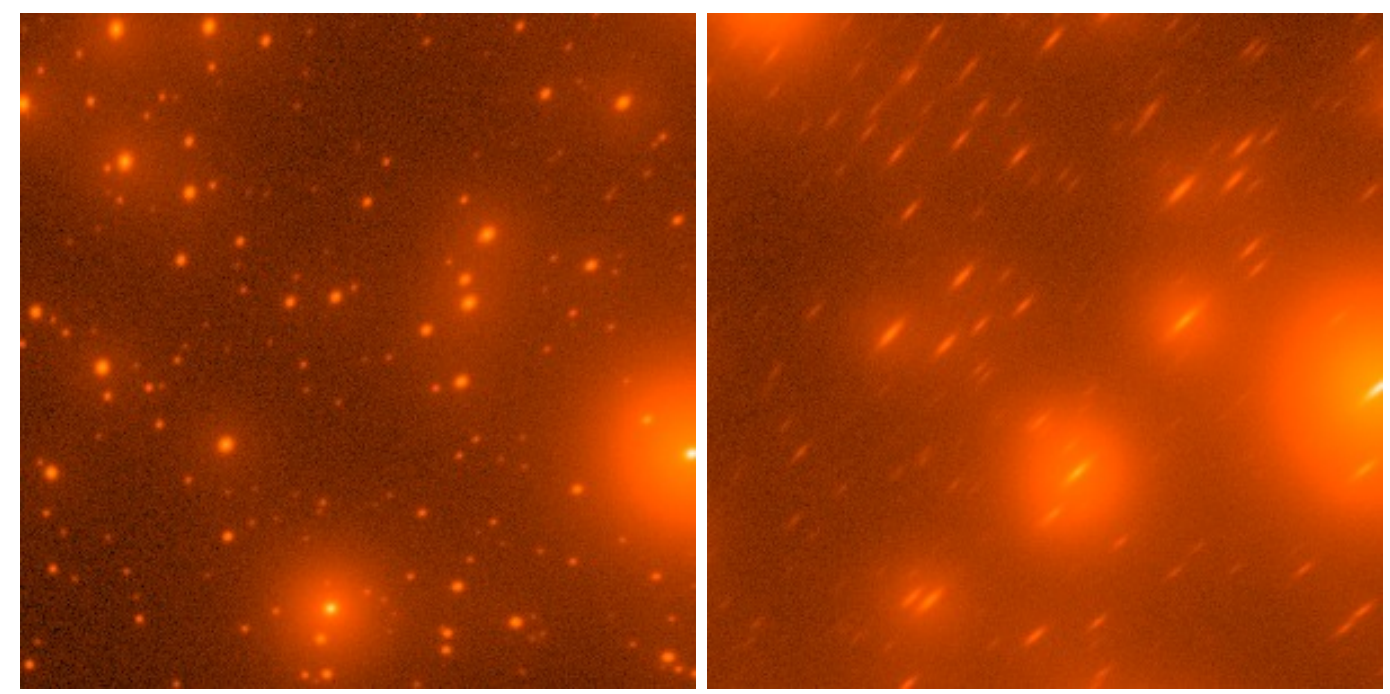

Figure 5. Two different regions of the simulated images representing the stellar field. Left panel: a $\sim 6$ arcsec ${ }^{2}$ sub-image

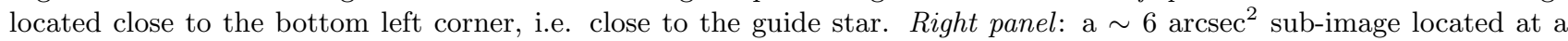
distance of $15^{\prime \prime}$.

elongation pattern pointing toward the GS. As shown in left panel of Fig. 4, the elongated half-light radius is variable across the FoV and its variation is described by a second degree polynomial function of the GS distance. The half-light radius pointing orthogonally to this direction has been considered constant and close to the diffraction limit.

- Seeing Halo: round Moffat (no elongation). The radius of this component has been set in order to reproduce a seeing disk of $\sim 0.6^{\prime \prime}$ in $J$ band. Being the total flux of the PSF model normalized to unitary volume, the only variable parameter in the $\mathrm{FoV}$ is the relative flux $F_{\text {Halo }}=1-F_{\text {Core }}$, where $F_{\text {Core }}$ is the relative flux contained in the Core component. The Halo component contains the signal due to the residual non corrected atmospheric aberrations and, therefore, its relative flux grows with the GS distance following a second order polynomial trend (Fig. 4, right panel).

For both the science cases, we placed the GS in the bottom left corner, just outside the FoV (pixel coordinates $[0,0]$ ). This choice allows us to maximize the PSF variation across a moderate FoV. In the same philosophy of enhancing the PFS variation, is the choice of a relative small isoplanatic angle of $\theta_{0} \sim 15^{\prime \prime}$ in $J$ band, leading to a maximum Strehl Ratio $<15 \%$ that rapidly decrease with the distance from the GS, reaching values close to zero at the image corner opposite to the GS.

The simulation of the AO corrected image is then the convolution of the true object with the local PSF + an additive zero mean random noise. In the case of the stellar field, the true object is represented by a set of simple delta-function sources weighted for their flux and with no noise. In the case of the Extragalactic field, we used a real high resolution image of a galaxy at z 0.1 obtained with HST.

\subsection{Stellar (point-like) sources}

We have simulated the observation of an external region of a 12 Giga-years old Galactic Globular Cluster (distance $=10 \mathrm{kpc}$ ). The selected region, containing $\sim 2800$ stars having $J \leq 25 \mathrm{mag}$, is located at a distance of $\sim 128^{\prime \prime}(=6.2 \mathrm{pc}$ ) from cluster center. The main parameters (magnitudes and colors) of the population forming the cluster have been drawn from theoretical models ${ }^{26}$ and the positions of the synthetic member stars have been computed from N-body realizations of an equilibrium King model. Fig. 5 shows two different regions of the simulated stellar field: the first one, close to the GS (left panel), presents round and peaked sources; the second one (right panel), located on the other side of the field, at a distance of about $15^{\prime \prime}$ from the GS, presents evidently elongated sources. In both sub-images it is easy to recognize the two Moffat components of the PSF: the variable diffraction limited core (with elongation pattern pointing toward the GS) and the constant seeing 

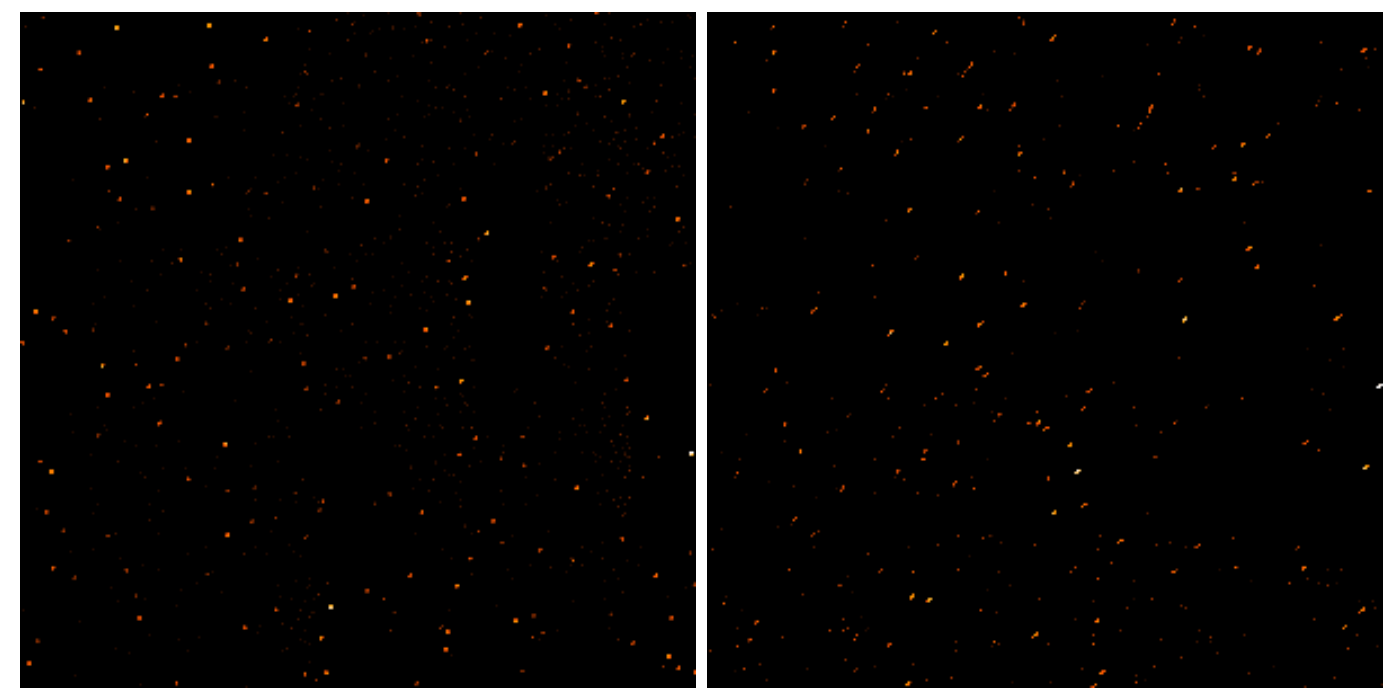

Figure 6. Example of reconstructed images when a grid of $15 \times 15$ PSFs at the center of their relative sub-domains are given to the Software Patch. The two panels refer to the same sub-images of Fig. 5

limited halo that surrounds all the sources. Because of its simplicity, the stellar case represents an interesting test-bench for the space-variant deconvolution algorithm. It permits to make an analysis on the reconstructed images depending on the PSF knowledge accuracy (i.e. on the density of the PSF grid) simply counting the sources in the object and in the reconstructed image.

We have run Patch on the simulated image using different PSF grids. In particular we gave as input to the program the PSFs at the center of $3 \times 3,5 \times 5,9 \times 9$ and $15 \times 15$ sub-domains. The central PSF of each sub-domain has been computed using the same analytical model that we used for the image simulation. Fig. 6 shows the result of the deconvolution in the same image regions shown in Fig. 5 when a grid of $15 \times 15$ PSFs is used. It is apparent that the reconstructed sources look similar to delta-functions and that the crowding effect due to the large amount of signal contained in the extended halos is disappeared. We recall that, by definition, the background of the reconstructed image is $=0$. Aperture photometry can be therefore performed even in presence of highly crowded fields and using relatively small apertures (few pixels). The sources in the right panel, that refers to the image region more affected by the elongated PSF shape, still presents a slight elongation in the direction of the GS position, especially at the sub-image edge. This is due to the PSF grid discretization, i.e. to the mismatch between the local PSF and the PSF at the sub-domain center. This residual elongation is anyway restrained (2-3 pixels) and can be easily contained in a small aperture.

After deconvolution algorithm has been applied, the restored image may include apparent artifacts such as dotting, striping or ringing. The artifacts are spurious deconvolution products and can be caused by different factors, like noise spikes or non-perfect knowledge of the PSF. Part of them can be identified simply defining a threshold map in the space of the reconstructed image. This map can be computed by dividing the noise map of the image before deconvolution by the maximum of the local normalized PSF. This number represents, point by point, the flux of the sources at the detection limit in the image space. All the pixels in the reconstructed image having a value lower than $\mathrm{n}$ * threshold (with $\mathrm{n}=1,2,3 \ldots$ depending on the desired confidence level) can be therefore labeled as 'non-detectable', and so, artifacts. The remaining artifacts are not distinguishable from the real sources. When more than an image is available, a match between different catalogues can filter out all the remaining spurious objects. In general, a good recipe to reduce as much as possible the number of artifacts is to choose an appropriate number of iterations and sub-domains and to try to extract the best possible PSF in each sub-domain.

In order to quantify how many sources have been reconstructed by the deconvolution process, we have analyzed the reconstructed image in a small region around the input sources coordinates, independently of their magnitude (no detection has been performed). We want to remark that the input sources coordinates are not integer pixel multiples, and so the resulting quasi-delta-functions are spread on more than 1 pixel. Table 1 collects 
Table 1. Percentage of lost sources in the reconstructed image within a certain aperture. The percentage refer to the total number of stars contained in the input list (2800) including the faintest ones.

\begin{tabular}{||cc||}
\hline $\begin{array}{c}\text { Number of } \\
\text { sub-domains }\end{array}$ & $\begin{array}{c}\text { Lost } \\
\text { objects }\end{array}$ \\
\hline $3 \times 3$ & $23 \%$ \\
$5 \times 5$ & $17 \%$ \\
$9 \times 9$ & $13 \%$ \\
$15 \times 15$ & $12 \%$ \\
\hline
\end{tabular}

Table 2. $50 \%$ and $80 \%$ completeness when considering all the reconstructed stars after deconvolution. The symbol '-' is reported when the completeness is always higher. The magnitude bin we considered is 0.1 mag. The faintest star of the input catalogue has a $J$ magnitude of 25.035 .

\begin{tabular}{||ccc||}
\hline $\begin{array}{c}\text { Number of } \\
\text { sub-domains }\end{array}$ & \multicolumn{2}{c|}{ Completeness } \\
\hline $3 \times 3$ & $80 \%$ \\
$5 \times 5$ & $24.74 \mathrm{mag}$ & $23.42 \mathrm{mag}$ \\
$9 \times 9$ & $24.95 \mathrm{mag}$ & $24.28 \mathrm{mag}$ \\
$15 \times 15$ & - & $24.25 \mathrm{mag}$ \\
\hline
\end{tabular}

the percentages of objects that 'disappear' in the reconstructed image and that cannot therefore be detected. In this table we defined 'lost' a star when the total counts within a certain aperture in the reconstructed image is $=0$. For this purpose we considered a 3 pixels diameter aperture.

Another interesting number is represented by the photometric depth at which the $50 \%$ or the $80 \%$ of the input objects at that magnitude are 'detected'. These values for the analyzed cases are reported in Table 2 . This Table confirms the fact that the lost stars of Table 1 are mainly faint stars. The photometric accuracy of the reconstructed sources varying the number of sub-domains is shown in Fig. 7. The photometric error has been computed comparing the true magnitude of the detected stars, with the one measured from the reconstructed image. As already mentioned, we performed a simple aperture photometry with relative small apertures. The aperture diameters have been set different for each case, being the residual elongation of the sources dependent on the PSF approximation. The aperture diameters we adopted are 13, 11, 7 and 5 pixels from the widest to the finest PSF grid step. Fig. 7 shows clearly an improvement of the reconstruction accuracy in terms of flux with the knowledge of the PSF. It is also apparent that the $9 \times 9$ and the $15 \times 15$ cases are almost equivalent in terms of number of retrieved objects and photometric accuracy. We have investigated this apparent saturation of the PSF knowledge repeating the experiment using intermediate numbers of sub-domains, confirming that in this case, using this PSF model and considering this variation across the FoV, more than $9 \times 9$ sub-domains do not improve the quality of the reconstructed image. As a term of comparison, in one case we have performed PSF fitting photometry using the last version of STARFINDER ${ }^{27}$ (V1.8.2a released in May 2014 ${ }^{25}$ ). STARFINDER analyzes the image considering local PSF estimates in different sub-domains. We used the $15 \times 15$ PSF grid. We do not deepen the comparison between the two results because the two software do basically two different things and give two different outputs: STARFINDER analyzes the image and produces stars catalogues; Patch is an image restoration software that produces another image. However a comparison with the photometric error in a given magnitude bin obtained with a largely diffused software, is a useful 'litmus paper' for the reliability of our software. Using STARFINDER we obtained a photometric error of $\sigma \sim 0.1 \mathrm{mag}$ at $21.5<J<22.5 \mathrm{mag}$, in a perfect agreement with the result obtained using Patch + simple aperture photometry. Furthermore, in our case seems to always exist an aperture that almost null the difference between the flux measured using the PSF fitting procedure and the aperture photometry. To conclude, we want to remark that the combination of the chosen PSF model, quickly variable across the FoV and inside each sub-domain, and of the GS position, at the edge of the FoV, makes the analyzed case quite conservative.

\subsection{Extragalactic extended source}

In this section we tested the capability of the Software Patch in the analysis of the morphological properties of extended sources. As starting object (true object), we used a real galaxy at $\mathrm{z} \sim 0.1$ observed at high resolution with HST. The flux in the central pixel of the galaxy has been increased by a factor 2 in order to simulate the 

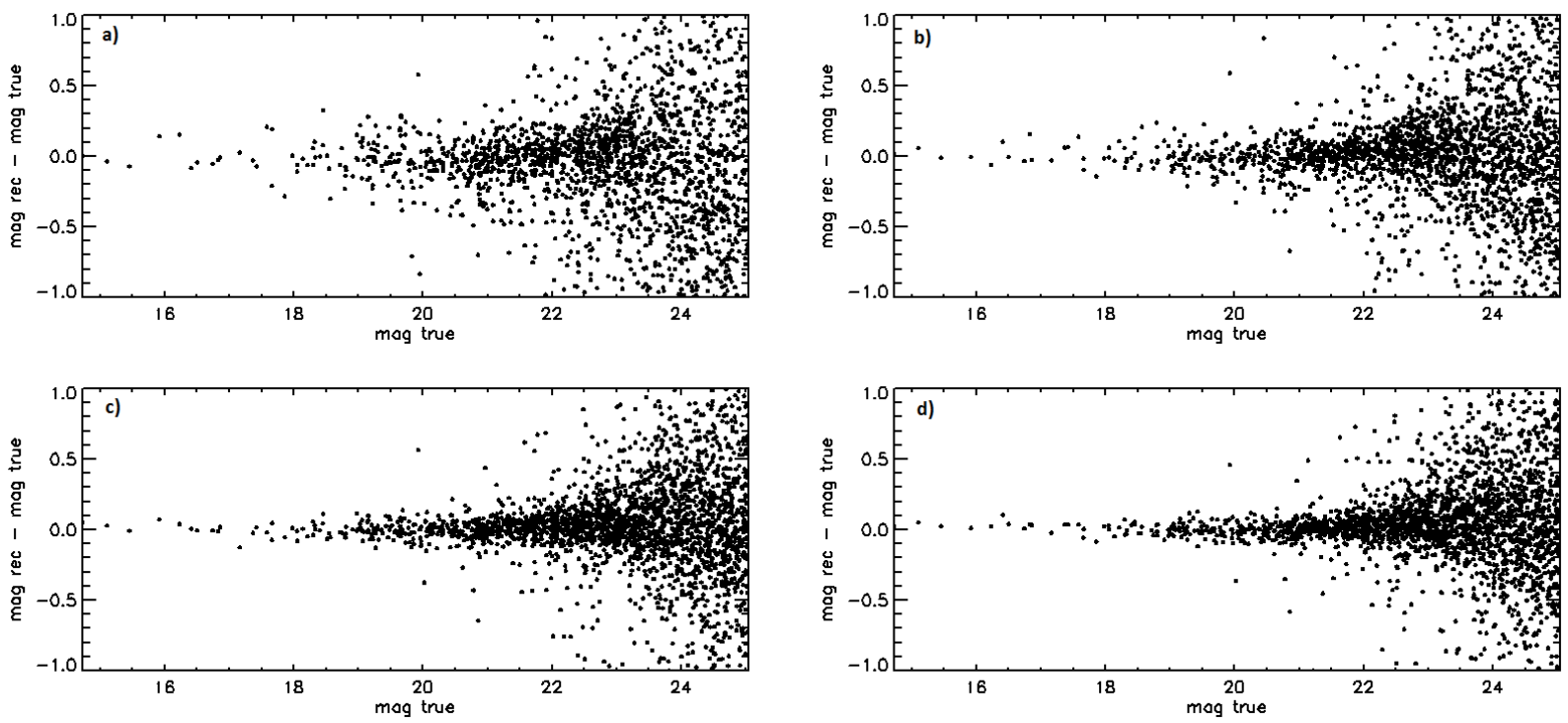

Figure 7. Comparison of the input and measured magnitudes of the detected stars (photometric error) for the analyzed cases: a) $3 \times 3$ sub-domains; b) $5 \times 5$ sub-domains; c) $9 \times 9$ sub-domains; d) $15 \times 15$ sub-domains. At $J \sim 22$ mag, a photometric error of $\sigma \sim 0.15,0.13,0.11$ and $0.09 \mathrm{mag}$ is reached for the cases a), b), c) and d) respectively.

presence of an Active Galactic Nuclei (AGN). We placed this galaxy in the three different places, with the centre of the galaxy at a distance of $7^{\prime \prime}, 14^{\prime \prime}$, and $21^{\prime \prime}$ from the bottom left corner, where the GS for the AO correction has been placed and then we convolved this image with the space variant PSF (see Sections 3.1).

The simulated image has a pixel size of $0.02^{\prime \prime}$ / pixel, while the galaxy has a total extension of 160 pixels (diameter) corresponding to $3.2^{\prime \prime}$ and a nucleus of 20 pixels in diameter (corresponding to $0.4^{\prime \prime}$ ). The simulated galaxy at three different distance from the GS for the AO correction is shown in Fig. 8. Image quality degradation due to the PSF variation is evident from left to right panels.

Using the same procedure adopted for the stellar case, we have run Patch on the simulated image using different PSF grids of $3 \times 3,5 \times 5,9 \times 9$ and $15 \times 15$ sub-domains. Fig. 9 shows the result of the deconvolution when a grid of $15 \times 15$ PSFs is used. The capability of the Software Patch in the imaging reconstruction for extended sources has been tested using the half-light radius $\mathrm{r}_{50}$, the concentration index $C_{28}$ and the light profile on the whole galaxy. The concentration index measures the concentration of flux about a central point in the galaxy. While slightly different version have been introduced by various author, we adopted the $C_{28}$ standard value $^{28}: C_{28}=5 \log \left(r_{80} / r_{20}\right)$ where $r_{20}$ and $r_{80}$ are the circular radii containing $20 \%$ and $80 \%$ of the total flux. As reference images, we used the original HST image, assumed to be the true objets before the PSF degradation, the simulated space-variant PSF image (assumed to be the observed image, see Fig. 8) and the reconstructed image after the application of Software Patch (see Fig. 9).

The results of our analysis are reported in Table 3 and Table 4, where the half-light radius $\mathrm{r}_{50}$ (in pixels) and the concentration index $C_{28}$ are reported for the 3 reference images used and for four different reconstructed images obtained using different PSF grids of sub-domains. As shown in the two Tables, in the observed image there is a clear degradation of the image quality, both in terms of the half-light radius $\mathrm{r}_{50}$ and concentration index $C_{28}$ with increasing distance from the GS for the AO correction (trend that is clearly visible also in Fig. 8). With the Software Patch we obtained a good reconstruction of the original image, even at large distance from the GS, although some artifacts produced by the deconvolution process are clearly visible in the image (see Fig. 9).

Finally, similar results have been obtained when we considered the light profile of the galaxy. In Fig. 10 we show the light profile for the whole galaxy for the three different images considered (real image (blue line), observed image (red line) and reconstructed image (green line). The three panels from left to right show the light profiles at increasing distance from the GS : 7, 14 and 21 arcsec respectively. 
Table 3. The half-light radius $\mathrm{r}_{50}$ (in pixel) and the concentration index $C_{28}$ for the galaxy simulated at different distance from the GS. The True Object is the original HST image while the Observed Image is the simulated space-variant PSF image.

\begin{tabular}{||cc||cccccc||}
\hline \multicolumn{1}{|c||}{ True Object } & \multicolumn{8}{c||}{ Observed Image } \\
& & \multicolumn{2}{c||}{ GS at $7^{\prime \prime}$} & GS at $14^{\prime \prime}$ & GS at $21^{\prime \prime}$ \\
$\mathrm{r}_{50}$ & $C_{28}$ & $\mathrm{r}_{50}$ & $C_{28}$ & $\mathrm{r}_{50}$ & $C_{28}$ & $\mathrm{r}_{50}$ & $C_{28}$ \\
\hline \multirow{3}{*}{33.10} & 2.78 & 36.50 & 2.50 & 38.20 & 2.37 & 39.20 & 2.30 \\
\hline
\end{tabular}

Table 4. The half-light radius $\mathrm{r}_{50}$ (in pixel) and the concentration index $C_{28}$ for the four reconstructed images with different PSF grids of sub-domains.

\begin{tabular}{||ccccccc||}
\hline \multicolumn{8}{|c}{ Reconstructed Image } \\
Number of & GS at $7^{\prime \prime}$ & GS at $14^{\prime \prime}$ & GS at $21^{\prime \prime}$ \\
sub-domains & $\mathrm{r}_{50}$ & $C_{28}$ & $\mathrm{r}_{50}$ & $C_{28}$ & $\mathrm{r}_{50}$ & $C_{28}$ \\
\hline $3 \times 3$ & 33.90 & 2.68 & 33.10 & 2.78 & 32.80 & 2.80 \\
$5 \times 5$ & 32.60 & 2.85 & 33.10 & 2.78 & 33.20 & 2.75 \\
$9 \times 9$ & 32.90 & 2.81 & 33.20 & 2.78 & 33.20 & 2.77 \\
$15 \times 15$ & 33.30 & 2.76 & 33.10 & 2.78 & 33.20 & 2.78 \\
\hline
\end{tabular}
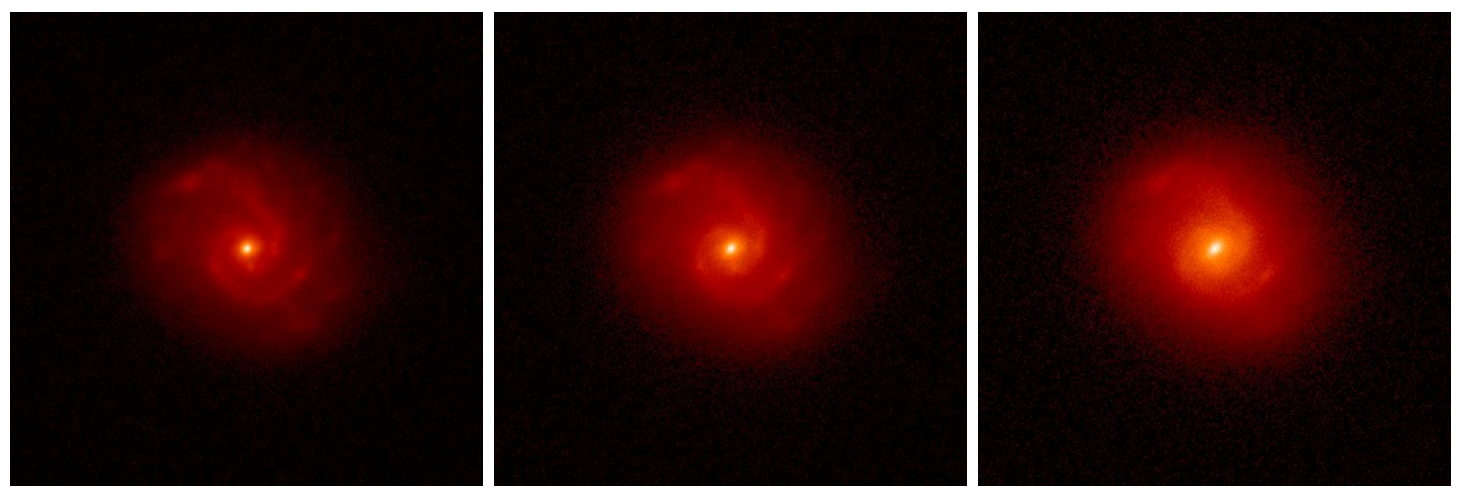

Figure 8. The simulated galaxy at increasing distance from the GS used for the AO correction: $7^{\prime \prime}$ (left panel), 14" (central panel) and $21^{\prime \prime}$ (right panel). Image quality degradation due to the PSF variation is evident from left to right panels.
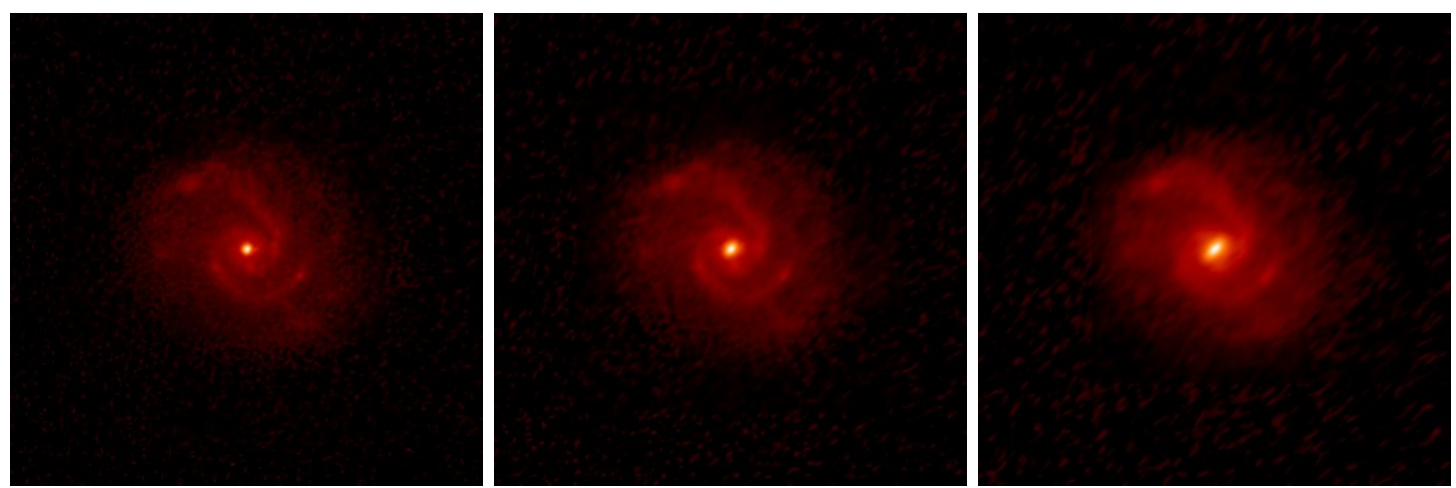

Figure 9. The reconstructed galaxy (with a grid of $15 \times 15$ PSFs) at increasing distance from the GS used for the AO correction: $7^{\prime \prime}$ (left panel), 14" (central panel) and $21^{\prime \prime}$ (right panel). 

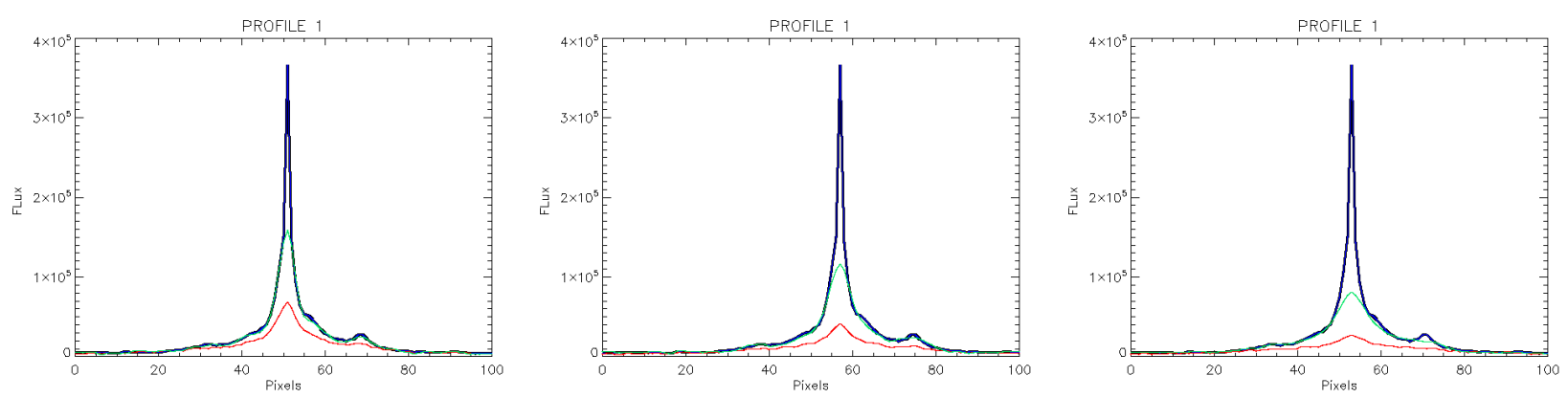

Figure 10. Light profile for the galaxy at $7^{\prime \prime}$ from GS (left panel), at $14^{\prime \prime}$ from GS (central panel) and at $21^{\prime \prime}$ from the GS (right panel). The blue solid line shows the light profile of the HST images assumed to be the real image, the red solid line shows the simulated observed image while the green solid line shows the image reconstructed with the software PATCH

\section{CONCLUSIONS AND FUTURE WORKS}

We developed a new method and software for the reconstruction of astronomical images with a space variant PSF. The software, called Patch, is written in IDL and can be freely downloaded from the software section of the website http://www.airyproject.eu. An interesting application is represented by AO corrected images that are characterized by structured and variable PSF. The potentiality of the Software Patch has been tested on two completely different astrophysical scenarios: a crowded stellar field and an extended galaxy. For both scenarios the images have been simulated assuming a PSF strongly variable across the FoV and a GS for the AO correction at the edge of the FoV, far away from the astronomical targets. Despite these very conservative assumptions, we obtained good results in terms of image reconstruction both for the stellar (point-like) case and for the extended galaxy.

However, we would like to stress that these results have been obtained using an input PSF grid computed with the same model used to generate the image. A more realistic simulation can be obtained using, as input for the deconvolution process, a PSF directly estimated from the image or from the AO loop data. The field of the $\mathrm{PSF}$ extraction/reconstruction is a very active and important field in the AO era, with several groups working on the extraction of the PSF directly from the image ${ }^{25,29}$ and on the reconstruction of long exposure PSF from the AO loop data ${ }^{3031}$. A strong synergy between the development of new deconvolution methods and the new techniques for the PSF extraction/reconstruction is very important in the AO era, when images with complex, space variant PSF will be the ordinary images for the next generation telescopes.

On the basis of these results, our next steps will be to take into account real astronomical images. The analysis with the Software Patch of a Globular Cluster observed with different AO facilities and of several galaxies at $\mathrm{z} \sim 1$ observed with HST is currently in progress.

\section{ACKNOWLEDGMENTS}

This research has been funded by the PRIN TECNO INAF 2010 : Exploiting the adaptive power: a dedicated free software to optimize and maximize the scientific output of images from present and future adaptive optics facilities. and by the Progetto Premiale E-ELT 2012 (PI Monica Tosi) funded by the Italian Ministry for Education, University and Research. L.S. acknowledges Antonio Sollima for useful discussions.

\section{REFERENCES}

[1] Gilmozzi, R. and Spyromilio, J., "The 42m European ELT: status," in [Society of Photo-Optical Instrumentation Engineers (SPIE) Conference Series], Society of Photo-Optical Instrumentation Engineers (SPIE) Conference Series $\mathbf{7 0 1 2}$ (Aug. 2008).

[2] Simard, L., Crampton, D., Ellerbroek, B., and Boyer, C., "The instrumentation program for the Thirty Meter Telescope," in [Society of Photo-Optical Instrumentation Engineers (SPIE) Conference Series], Society of Photo-Optical Instrumentation Engineers (SPIE) Conference Series 8446 (Sept. 2012). 
[3] Johns, M., McCarthy, P., Raybould, K., Bouchez, A., Farahani, A., Filgueira, J., Jacoby, G., Shectman, S., and Sheehan, M., "Giant Magellan Telescope: overview," in [Society of Photo-Optical Instrumentation Engineers (SPIE) Conference Series], Society of Photo-Optical Instrumentation Engineers (SPIE) Conference Series 8444 (Sept. 2012).

[4] Beckers, J. M., "Increasing the size of the isoplanatic patch with multiconjugate adaptive optics," in [ESO Conference on Very Large Telescopes and their Instrumentation], Ulrich, M.-H., ed., ESO Conference and Workshop Proceedings, No. 30 2, 693-703 (1988).

[5] Marchetti, E., Brast, R., Delabre, B., Donaldson, R., Fedrigo, E., Frank, C., Hubin, N., Kolb, J., Lizon, J.-L., Marchesi, M., Oberti, S., Reiss, R., Soenke, C., Tordo, S., Baruffolo, A., Bagnara, P., Amorim, A., and Lima, J., "MAD on sky results in star oriented mode," in [Society of Photo-Optical Instrumentation Engineers (SPIE) Conference Series], Society of Photo-Optical Instrumentation Engineers (SPIE) Conference Series 7015 (July 2008).

[6] Rigaut, F., Neichel, B., Boccas, M., d'Orgeville, C., Arriagada, G., Fesquet, V., Diggs, S. J., Marchant, C., Gausach, G., Rambold, W. N., Luhrs, J., Walker, S., Carrasco-Damele, E. R., Edwards, M. L., Pessev, P., Galvez, R. L., Vucina, T. B., Araya, C., Gutierrez, A., Ebbers, A. W., Serio, A., Moreno, C., Urrutia, C., Rogers, R., Rojas, R., Trujillo, C., Miller, B., Simons, D. A., Lopez, A., Montes, V., Diaz, H., Daruich, F., Colazo, F., Bec, M., Trancho, G., Sheehan, M., McGregor, P., Young, P. J., Doolan, M. C., van Harmelen, J., Ellerbroek, B. L., Gratadour, D., and Garcia-Rissmann, A., "GeMS: first on-sky results," in [Society of Photo-Optical Instrumentation Engineers (SPIE) Conference Series], Society of Photo-Optical Instrumentation Engineers (SPIE) Conference Series 8447 (July 2012).

[7] Diolaiti, E., "MAORY: A Multi-conjugate Adaptive Optics RelaY for the E-ELT," The Messenger 140, 28-29 (June 2010).

[8] La Camera, A., Schreiber, L., Diolaiti, E., Boccacci, P., and Bertero, M., "A method for space-variant deblurring with application to adaptive optics imaging in astronomy," in preparation .

[9] Trussel, H. J. and Hunt, B. R., "Sectioned methods for image restoration," IEEE Trans. Acoustics, Speech and Signal Proc. 26, 157-164 (1978a).

[10] Trussel, H. J. and Hunt, B. R., "Image restoration of space-variant blurs by sectioned methods," IEEE Trans. Acoustics, Speech and Signal Proc. 26, 608-609 (1978b).

[11] Boden, A. F., Redding, D. C., Hanisch, R. J., and Mo, J., "Massively parallel spatially-variant maximum likelihood restoration of hubble space telescope imagery," J. Opt. Soc. Am. A-13, 15337-1545 (1996).

[12] Aubailly, M., Roggermann, M. C., and Schulz, T. J., "Approach for reconstructing anisoplanatic adaptive optics images," Appl. Optics 46, 6055-6063 (2007).

[13] Prato, M., Cavicchioli, R., Zanni, L., Boccacci, P., and Bertero, M., "Efficient deconvolution methods for astronomical imaging: algorithms and IDL-GPU codes," Astron. Astrophys. 539, A133 (2012).

[14] Richardson, W. H., "Bayesian based iterative method of image restoration," J. Opt. Soc. Am. 62, 55-59 (1972).

[15] Lucy, L. B., "An iterative technique for the rectification of observed distributions," Astron. J. 79, 745-754 (1974).

[16] Bonettini, S., Zanella, R., and Zanni, L., "A scaled gradient projection method for constrained image deblurring," Inverse Problems 25(1), 015002 (2009).

[17] Bertero, M., Boccacci, P., Desiderá, G., and Vicidomini, G., "Image deblurring with Poisson data: from cells to galaxies," Inverse Problems 25, 123006 (2009).

[18] Bertero, M. and Boccacci, P., "A simple method for the reduction of boundary effects in the Richardson-Lucy approach to image deconvolution," Astron. Astrophys. 437, 369-374 (2005).

[19] Anconelli, B., Bertero, M., Boccacci, P., Carbillet, M., and Lantéri, H., "Reduction of boundary effects in multiple image deconvolution with an application to LBT LINC-NIRVANA," Astron. Astrophys. 448, 1217-1224 (2006).

[20] Bertero, M., Boccacci, P., Talenti, G., Zanella, R., and Zanni, L., "A discrepancy principle for poisson data," Inverse Problems 26, 10500 (2010). 
[21] Fusco, T., Mugnier, L. M., Conan, J.-M., Marchis, F., Chauvin, G., Rousset, G., Lagrange, A.-M., Mouillet, D., and Roddier, F. J., "Deconvolution of astronomical images obtained from ground-based telescopes with adaptive optics," in [Adaptive Optical System Technologies II], Wizinowich, P. L. and Bonaccini, D., eds., Society of Photo-Optical Instrumentation Engineers (SPIE) Conference Series 4839, 1065-1075 (Feb. 2003).

[22] Lauer, T., "Deconvolution with a spatially-variant PSF," in [Astronomical Data Analysis II], Starck, J.-L. and Murtagh, F. D., eds., Society of Photo-Optical Instrumentation Engineers (SPIE) Conference Series 4847, 167-173 (Dec. 2002).

[23] Guerra, J. C., Rakich, A., Green, R., McCarthy, D., and Kulesa, C., "PISCES Infrared Imager Performance with the Large Binocular Telescope Adaptive Optics System," tech. rep., Large Binocular Telescope Observatory and Steward Observatory (2013).

[24] Pedani, M., "Sky surface brightness at Mount Graham II. First JHKs science observations with the Large Binocular Telescope," New Astronomy 28, 63-69 (Apr. 2014).

[25] Schreiber, L., Diolaiti, E., Bellazzini, M., Ciliegi, P., Foppiani, I., Greggio, L., Lanzoni, B., and Lombini, M., "Handling a highly structured and spatially variable Point Spread Function in AO images," in [Second International Conference on Adaptive Optics for Extremely Large Telescopes. Online at iA href="http://ao4elt2.lesia.obspm.fr" ihttp://ao4elt2.lesia.obspm.fri/Ai், id.P57], 57P (Sept. 2011).

[26] Dotter, A., Chaboyer, B., Jevremović, D., Kostov, V., Baron, E., and Ferguson, J. W., "The Dartmouth Stellar Evolution Database," The Astrophysical Journal Supplement 178, 89-101 (Sept. 2008).

[27] Diolaiti, E., Bendinelli, O., Bonaccini, D., Close, L., Currie, D., and Parmeggiani, G., "Analysis of isoplanatic high resolution stellar fields by the StarFinder code," A\& A 147, 335-346 (Dec. 2000).

[28] Law, D. R., Steidel, C. C., Shapley, A. E., Nagy, S. R., Reddy, N. A., and Erb, D. K., "A HST/WFC3IR Morphological Survey of Galaxies at $\mathrm{z}=1.5-3.6$. II. The Relation between Morphology and Gas-phase Kinematics," ApJ 759, 29 (Nov. 2012).

[29] Schreiber, L., Diolaiti, E., Sollima, A., Arcidiacono, C., Bellazzini, M., Ciliegi, P., Falomo, R., Foppiani, I., Greggio, L., Lanzoni, B., Lombini, M., Montegriffo, P., Dalessandro, E., and Massari, D., "Developing a new software package for PSF estimation and fitting of adaptive optics images," in [Society of Photo-Optical Instrumentation Engineers (SPIE) Conference Series], Society of Photo-Optical Instrumentation Engineers (SPIE) Conference Series $\mathbf{8 4 4 7}$ (July 2012).

[30] Veran, J.-P., Rigaut, F., Maitre, H., and Rouan, D., "Estimation of the adaptive optics long-exposure point-spread function using control loop data.," Journal of the Optical Society of America A 14, 3057-3069 (Nov. 1997).

[31] Fusco, T., Conan, J.-M., Mugnier, L. M., Michau, V., and Rousset, G., "Characterization of adaptive optics point spread function for anisoplanatic imaging. Application to stellar field deconvolution," $A \& \mathcal{E} A \mathbf{1 4 2}$, 149-156 (Feb. 2000). 